\title{
Diseño de packaging ecológico para producto en polvo soluble
}

\author{
Design of Ecologic Packaging for \\ a Product in Soluble Powder
}

\author{
Erika Böhm Silveti (España) \\ Institución Universitaria Politécnico Grancolombiano \\ Máster en Ingeniería y Gestión del Medio Ambiente.
} ebs1980@hotmail.com

Aida Ximena León Guatame (Colombia) Institución Universitaria Politécnico Grancolombiano Magister en Pedagogía y Profesional en Mercadeo.

aleongua@poligran.edu.co

\section{Resumen \\ Este artículo entrega alternativas de diseños de packaging ecológicos para un producto denominado Cosecha: frutas y verduras en polvo soluble, mediante proceso de atomización que, según el experto consultado, es un proceso que permite conseguir un tamaño de partículas apropiado para que sea soluble y al mismo tiempo conserve las propiedades originales de los alimentos durante 6 meses en}

\begin{abstract}
This article contains alternative ecological packaging designs for a product called Cosecha: fruits and vegetables in soluble powder. This is carried out through an atomization process that, according to the expert Professor Povea (from the Department of Food Engineering of La Salle University), allows obtaining the appropriate size of particles in order for them to be soluble and at the same time maintain the original properties of food for six months
\end{abstract}

$\begin{array}{lrl}\text { RECIBIDO: } & 23 \text { de febrero de } 2015 & \text { PARA CITAR ESTE ARtículo / to CITE thiS ARTICLE } \\ \text { EVALUADO: } & 6 \text { de abril de } 2015 & \text { Böhm Silveti, E. y león Guatame, A. X. (2015). Dise- } \\ \text { ACEPTADO: } & 19 \text { de mayo de } 2015 & \text { ño de packaging ecológico para producto en polvo } \\ & \text { soluble. Poliantea, } 77(20) \text {. pp.67-86. }\end{array}$

POLIANTEA | P. 67-86 | VOLUMEN XI | NÚMERo 20| ENERO-JUNio 2015 
un envase apropiado. Este producto solucionaría el problema que existe en los bancos de alimentos de Bogotá, donde reposan gran cantidad de frutas y verduras que han sido desechadas por estar sobremaduradas, golpeadas, mal empacadas o devueltas por los almacenes de cadena (300 $\mathrm{mm} /$ día), que pueden ser aprovechadas para producir productos alimenticios procesados y susceptibles de comercializar. Sin embargo, es de destacar que el interés del estudio se centró en diseñar envases que respondieran a las tendencias que hay respecto de packaging, es decir que fuera ecológico, funcional, emocional, llamativo para la población bogotana de estratos 2 y 3 , además de responder a las necesidades del segmento. Los resultados obtenidos evidenciaron que el producto Cosecha: frutas y verduras en polvo soluble responde a una necesidad y hábito de la población y al mismo tiempo que las decisiones tomadas para el diseño de los envases fueron acertadas, de tal forma que la mayoría de los encuestados dijeron que los prototipos les resultan llamativos e innovadores y que comprarían el producto a un precio razonable bajo dos de los tres prototipos propuestos.

Palabras clave: packaging ecológico, materiales, diseño estructural, diseño gráfico, sellos verdes. in the appropriate container. This product would solve the problem that food banks in Bogotá have regarding fruits and vegetables that are rejected because they are overripe, bruised, incorrectly packed, or returned by chain stores $(300 \mathrm{~mm} /$ day) because they could be used to produce processed food that can be commercialized. However, it is important to indicate that the study aimed to design containers that respond to the current tendencies with respect to packaging. This means, something ecological, functional, emotional, and appealing for people that belong to the middle low class (strata 2 and 3 in Colombia) besides responding to the needs of the segment. The results evidenced that the product Cosecha: fruits and vegetables in soluble powder respond to a need and a habit of the population and with its appropriate container design, it was possible to obtain positive feedback from the majority of the respondents. They said that they thought the containers were innovative and appealing and indicated they would be willing to buy the product as long as it is affordable (two of the three prototypes proposed.)

Keywords: ecological packaging materials, structural design, graphic design, green seal 


\section{Introducción}

El producto Cosecha: frutas y verduras en polvo soluble pretende ofrecerse en un packaging que sea amigable con el medio ambiente para ser ofrecido a personas que habiten en Bogotá en estratos 2 y 3 . Este estudio se centró en conocer las teorías sobre empaques ecológicos aptos para frutas y se apoyó en expertos para determinar el diseño estructural y gráfico de envases que motiven la compra del producto por parte de personas de estratos 2 y 3 de Bogotá, ya que representan $80 \%$ de la población (DANE, 2013).

Se consultaron estudios, como los realizados por Arena Media de la empresa Havas Media Group (2011), en los que se caracteriza al consumidor colombiano de dichos estratos como personas tradicionales, que buscan bebidas solubles, fáciles de tomar, de tamaños individuales y familiares con sabores que evoquen al ayer, pero a quienes les gusta innovar de vez en cuando e influye el etiquetado y la semiótica de los envases a la hora de comprar (Pineda, 2011)

En cuanto a la funcionalidad, se tomó en cuenta el estudio de Yanhaas (2004) sobre el estilo de vida de los colombianos, que muestra una tendencia hacia la practicidad desde 2004, en la que todo lo que permita ahorrar tiempo y esfuerzo, como los productos en presentaciones portables, es cada vez más valorado y considerado como alternativas muy llamativas a la hora de la decisión de compra.

Asimismo el estudio realizado a 500 compradores por Tiendeo.com.co (Universia, 2014) sobre la importancia que tiene la protección del medio ambiente a la hora de tomar la decisión de compra de un cierto producto, el cual evidencia que para $80 \%$ de los encuestados es imporyante que fabricantes y distribuidores consideren el tema ambiental sin importar 
si el producto tiene un envase sostenible o no.

Por otra parte, hoy en día la mayoría de los productos alimenticios que se ofrecen en el mercado tienen fácilmente imitables entre empresas. De hecho, estos aspectos son rápidamente adaptados, mejorados o modificados por la competencia, tras lo cual crean un espacio limitado en el que sobresalir en las góndolas y llamar la atención del consumidor (Cajamarca y Murillo, 2011). A diferencia de la exposición publicitaria, que puede ser muy corta, el packaging en los productos alimenticios procesados sigue construyendo valores de marca después de la compra, es decir, durante su uso.

Respecto del diseño de envases, Colombia está empezando a despertar de un largo estancamiento, la industria se encuentra en posiciones inferiores con respecto a países, como México, Brasil, Chile y Argentina, en los que las empresas tanto productoras como consumidoras de materiales de envases tienen una mayor tradición en la exportación de productos transformados y sus economías dependen menos de productos primarios, como sucede en Colombia.

Sin embargo, es notorio el interés que en los últimos años se ha venido despertando entre los productores colombianos por mejorar los envases tanto en lo estético y de funcionalidad como en la calidad de los materiales y según estándares técnicos internacionales (Elempaque.com, s. f.).

Este despertar se debe a que la industria ha comprendido que el consumidor es cada vez más exigente y es quien determina en el punto de venta si un producto sobrevive a la dura competencia de la góndola o perece favoreciendo aquellos productos que han entendido los gustos y las necesidades de los compradores. Es decir, para conseguir estar dentro de las alternativas de compra y en la mente del consumir, es clave estar al día en sus necesidades funcionales y en las tendencias que marcarán el futuro de los envases. Dentro de las tendencias se encuentran los envases ecológicos, envases inteligentes, envases de convivencia, adaptados a cada consumidor, envases para pauta publicitaria y envases comestibles (Ardila, 2014).

\section{Objetivo general}

Reconocer las cualidades de packaging ecológico que motiven la compra del producto Cosecha: frutas $y$ verduras en polvo soluble mediante proceso de atomización en consumidores de estratos 2 y 3 de Bogotá. 


\section{Objetivos específicos}

- Especificar los materiales de empaque ecológico por utilizarse en el envase del producto frutas y verduras en polvo soluble mediante proceso de atomización de nombre Cosecha.

- Reconocer las características visuales y ergonómicas de los empaques ecológicos que motiven la compra del producto frutas y verduras en polvo mediante proceso de atomización de nombre Cosecha en consumidores de estratos 2 y 3 de Bogotá.

- Definir los elementos semióticos del empaque ecológico para transmitir los beneficios del producto frutas y verduras en polvo mediante proceso de atomización de nombre Cosecha en consumidores de estratos 2 y 3 de Bogotá.

\section{Marco teórico}

Hablar de empaques ecológicos resulta difícil desde el punto de la concepción agropecuaria; sin embargo, se realizó una revisión sobre estudios relacionados y su impacto en el medio ambiente.

De esta manera, según Aramendiz-Tatis et al. (2007), para almacenar semillas de berenjena se recomiendan bolsa de papel y frascos de vidrio, con la particularidad de que son perjudiciales para las semillas porque no protege de la humedad y temperatura. Las bolsas de papel blanco tratado no son ecológicas, los frascos de vidrio son reutilizables y reciclables, aunque tienen riesgos de ruptura, por lo que exigen cuidados en el almacenaje.

Según Flórez, Marín y Zapata (2009), el empaque adecuado para la papaya es el guacal de madera, canastillas plásticas y cajas de cartón. Hay que tener en cuenta que cuando la madera no proviene de la tala indiscriminada de bosques que provoca un impacto negativo en el medio ambiente, sino de bosques controlados, es un material renovable y reciclable.

Según García, Cury y Dussán (2011), para la guayaba se recomienda la bandeja de poliestireno cubierta con película plástica de policloruro de vinilo, que es un material biológicamente inerte, no tóxico y estable, puede reciclarse y reutilizarse. Sin embargo, la película plástica de policloruro de vinilo, al llevar cloro en su composición, como el DDT (dicloro difenil tricloroetano), durante su producción, su uso y cuando se quema forma sustancias organocloradas, extremadamente tóxicas para el medio ambiente y para la salud de 
las personas, además para su fabricación se utilizan metales pesados y cancerígenos, como el cadmio.

Según Cañón et al. (2014), para la feijoa, se recomienda un empaque de cartón corrugado, que, por no tener componentes tóxicos, su reciclaje es seguro y ahorra energía, recursos naturales y espacio necesario en los vertederos. Otro empaque recomendado es el conocido PET o alveolos de politereftalato, el tereftalato de polietileno, politereftalato de etileno, polietilentereftalato o polietileno tereftalato, que es un polímero obtenido con una reacción de policondensación entre el ácido tereftálico y el etilenglicol, perteneciente al grupo de materiales sintéticos denominados poliésteres, elaborado a base de combustibles fósiles, lo cual provoca una excesiva presión sobre las limitadas fuentes de energía no renovables, por lo que una vez que los plásticos se han utilizado se convierten en residuos sólidos urbanos, que generados en grandes cantidades producen problemas de contaminación de agua, aire y suelo, y afectan directamente al ambiente y a la salud.

Según Dussán et al. (2014), para el mango, las bolsas de polietileno, las bandejas de poliestireno cubiertas con policloruro de vinilo, como se indicó, no son ecológicas.
Diseñar un packaging ecológico implica diseñar de una forma más funcional, práctica, sencilla y mucho menos contaminante; es decir, desarrollar envases que ocupen menos espacio, se almacenen mejor, se fabriquen utilizando menos recursos (materias primas, energía...) y se transporten de forma más eficiente, de tal forma que se minimice el impacto que su existencia tiene en el medio ambiente (Martín, 2013).

Para que un envase se considere ecológico, debe poseer alguna de las siguientes cualidades:

Ser biodegradable, es decir que respete el medio ambiente y su descomposición se realice con la acción de agentes biológicos, bajo condiciones ambientales naturales; o tener una huella de $\mathrm{CO}_{2}$ baja, es decir, sus emisiones de carbono a la atmósfera tanto durante su producción como uso sea la más baja posible; o reciclable o reutilizable, lo cual significa que esté fabricado con materiales que una vez sean residuos puedan ser tratados para convertirlos en un material nuevamente utilizable o volverse a emplear para el mismo o un fin distinto.

$\mathrm{Al}$ mismo tiempo, el envase debe ser higiénico, cumpliendo con lo que se refiere a limpieza, esterilidad y conservación del producto, y 
comunicador, ya que es una herramienta clave de comunicación e información a través de la cadena logística hasta el consumidor final (Moinhos, García-Inés y Alberto, 2011).

Teniendo en cuenta lo anterior, cabe anotar que Colombia, según Richard Probst, gerente de la empresa Green Bizz Consulting, respecto del sector de bienes y servicios ecológicos en el país, se encuentra muy por debajo de su potencial, lo cual se refleja en aspectos de mercadeo y marketing, así como en la oferta y calidad de los envases, donde las técnicas para el mercado local no llegan al uso de materiales ecológicos y se siguen utilizando materiales convencionales derivados del petróleo.

Según Carolina Alzate, diseñadora de envases de la empresa IMA+D, este hecho ocurre porque el packaging ecológico ya no es una moda, sino una obligación, y debe responder a las necesidades de los clientes respecto de los tamaños familiares e individuales, es decir que tengan doble función, que sean emocionales o que generen sorpresa al utilizarlos.

Los materiales tradicionalmente más utilizados y que más petróleo y gas natural necesitan para su producción son el plástico, el papel y el cartón tratado y la madera.
Según Selke (1994), Colombia dispone de materiales ecológicos a su alcance para el producto Cosecha ${ }^{1}$ objeto de esta investigación, así como el poliácido láctico, que es un polímero constituido por moléculas de ácido láctico, con propiedades semejantes a las del tereftalato de polietileno y es biodegradable. Además, se obtiene del almidón de maíz, que se fermenta para obtener el monómero, puede imprimirse sin tratamiento superficial y utilizar para la conformación de envases o de films termoadhesivos para etiquetados (Auras et al., 2011). Asimismo, el papel de fibra de caña de azúcar reciclable está clasificado internacionalmente como amigo de la naturaleza, y es un producto puro y biodegradable, ya que se elabora con el subproducto de la caña de azúcar (pulpa virgen), que es inoloro y limpio, con lo que puede estar en contacto directo con alimentos para consumo humano. En la misma línea, se encuentra el cartón brik, que ha demostrado ser un envase robusto, resistente

Para el producto frutas y verduras en polvo soluble mediante proceso de atomización de nombre Cosecha, se pretende transformarlo en pulpa de fruta, posteriormente pasarla por un proceso de atomización o secado aerosol a fin de conseguir microgotas deshidratadas y solubles $y$, así, mantener las propiedades físico-químicas de los productos $y$, en algunos casos, llegar a mejorar sus propiedades. 
a los golpes, que ocupa un espacio mínimo en la distribución del envase vacío, que al presentarse en forma de paralelepípedo mejorará la comunicación y el diseño de etiquetados. En relación con el producto, lo protege del aire, la luz, las bacterias y se elabora a partir de fibras largas que aportan mayor resistencia y rigidez, procedentes de plantaciones con repoblación continua de especies autóctonas de Finlandia y Suecia (Revista Ambientum, 2003). Por otro lado, también se puede optar por el aluminio reciclado, que es uno de los materiales que mejor conservan las propiedades de los alimentos porque reducen su desperdicio y la generación de residuos al proporcionar protección total de la luz, el oxígeno y los microbios nocivos. Además, el uso de aluminio reciclado reduce la demanda de material virgen con la consecuente reducción de consumo de energía y de las emisiones de CO² (Salazar, 2011). Por último, se encuentra el vidrio reciclado que, sin lugar a dudas, es uno de los materiales más ecológicos a la hora de hacer envases, puesto que, al estar hecho de abundantes materias primas, su extracción es sencilla y además es ciento por ciento reciclable, lo cual permite usar los envases infinidad de veces. También los envases de vidrio son inmunes al contacto con alimentos, que a diferencia de otras materias primas, como el plástico, no permiten migraciones de monómeros y aditivos hacia el producto; sin embargo, los precios son costosos, tienen un peso superior y exigen un cuidado especial por ser más delicados, ya que pueden generar cortes o heridas en caso de rotura (Revista de Logística, s. f.).

\section{Método}

El presente estudio es de tipo exploratorio debido a que la revisión de la literatura reveló que existe un bajo número de artículos relacionados con materiales sobre empaques ecológicos y no se encuentran estudios relacionados con este tipo de empaques para un producto con las características del de Cosecha ${ }^{2}$. Es una investigación no experimental transversal en la que se recolectan datos en un solo momento. Como método de recolección de información, se realizaron entrevistas a profundidad con cuestionario semiestructurado a un experto en conservación del producto en polvo soluble y otro en diseño de empaques, concluyendo que hay tres prototipos de

2 Frutas y verduras en polvo soluble mediante proceso de atomización. 
envases, los cuales fueron sometidos a opinión de 51 personas de estratos 2 y 3 habitantes de Bogotá mediante cuestionario estructurado.

\section{Materiales}

El instrumento estructurado que se aplicó estuvo conformado por 20 preguntas, en las que se contemplaron preguntas dicotómicas, abiertas, opción múltiple y escala Likert. Su construcción se realizó en preguntas de identificación, filtro y aquellas que pretendían conocer el empaque ecológico que fuera llamativo, así como la semiótica apropiada para el segmento del mercado elegido.

El instrumento semiestructurado estuvo compuesto por 19 preguntas abiertas, las cuales indagaban la descripción del empaque ecológico y llamativo.

\section{Resultados}

Una vez consultados los expertos, se determinó que el producto Cosecha es un polvo soluble al que le puede afectar tanto la humedad como la luz del sol y las altas temperaturas. Se seleccionaron materiales, como papel de caña de azúcar reciclado junto con lámina de aluminio reciclado PLA y lámina de aluminio y aluminio reciclado solo, porque conservan correctamente el producto, son livianos, resistentes y moldeables. Esta última característica tiene estrecha relación con la funcionalidad que se le puede dar a un envase, como formas que faciliten su agarre y almacenamiento, pero sin perder de vista la normativa por cumplir. A partir de fuentes secundarias, se tuvo en cuenta que, para cumplir con la normativa colombiana, es importante poner en consideración las resoluciones: 683 de $2012^{3}$, 1506 de $2011^{4}, 224$ de $2007^{5}$ y 333 de $2011^{6}$. Para la normativa ecológica sobre envases, se contempló la Resolución 187 de $2006^{7}$. Por su parte,

3 Sobre requisitos sanitarios que deben cumplir los materiales, objetos, envases y equipamientos destinados a entrar en contacto con alimentos y bebidas para consumo humano (Invima, 2014).

4 Sobre los requisitos de rotulado o etiquetado que deben cumplir los aditivos que se emplean para la elaboración de alimentos para consumo (Invima, 2014).

5 Enmarca los requisitos mínimos que deben cumplir los envases de los productos agrícolas para consumo humano que se importen, se produzcan y se comercialicen en el territorio nacional (Invima, 2014).

6 Señala los requisitos de rotulado o etiquetado nutricional que deben cumplir los alimentos envasados para consumo humano.

7 Reglamento para la producción primaria, procesamiento, empacado, etiquetado, almacenamiento, certificación, importación, comercialización, y establece el Sistema de Control de Productos Agropecuarios Ecológicos (ICA, 2006). 
en el ámbito internacional existe la normativa ISO TC $122 \mathrm{SC}^{8}{ }^{8}$.

Además, se contempló la posibilidad de certificar el proceso o producto o envase ecológico con sellos verdes, herramienta visual que permite informar y avisar a los consumidores sobre qué productos han sido concebidos con una perspectiva ecológica y en qué grado afectan al medio ambiente, de modo que el consumidor sea capaz de identificar y comprar aquellos productos y servicios que más encajen con sus valores y necesidades. Entre ellos se encuentran en el ámbito nacional el sello ambiental colombiano ${ }^{9}$ y el sello ecológico para alimentos ${ }^{10}$. Por otra parte, se

8 La cual regula los diseños de packaging y el cuidado del medio ambiente en su elaboración, uso y fin de uso (ISO, 2005).

9 Se otorga a aquel producto que hace uso sostenible de los recursos naturales que emplea (materia prima e insumos), utiliza materias primas que no son nocivas para el ambiente, emplea procesos de producción que involucran menos cantidades de energía o que hacen uso de fuentes de energía renovables, o ambas, considera aspectos de reciclabilidad, reutilización o biodegradabilidad, usa materiales de envase, preferiblemente reciclable, reutilizable o biodegradable y en cantidades mínimas, emplea tecnologías limpias o que generan un menor impacto relativo sobre el ambiente e indica a los consumidores la mejor forma para su disposición final (Incontec Internacional, s. f.).

10 Promueve, diferencia y posiciona los productos ecológicos dentro de toda la cadena de productos alimenticios, generando confianza en los consumidores al adquirir un producto con las características deseadas, respaldado reconoce que existen a disposición símbolos visuales internacionales de envases sostenibles, como el de reusar, reducir y reciclar, que indican que los materiales con los que ha sido fabricado un producto pueden ser reciclados; el símbolo del punto verde, que denota que el envase debe tener un ciclo de recuperación y reciclaje que permita reaprovechar los materiales que en su momento se usaron para fabricarlo; y el símbolo Tidyman, figura humana que deposita un residuo en una papelera, con lo cual indica al consumidor que se responsabilice de deshacerse de este en un lugar adecuado (Twenergy, 2011).

Una vez realizado el diseño estructural del envase que cumplía con las normativas, se diseña la parte gráfica, como marca, logotipo, nombre y mensaje o verbal branding, color del envase y diseño de la información de la etiqueta. Para darse a conocer, es necesario tener un buen nombre y un logo llamativo, por lo que algunas recomendaciones son utilizar un nombre que haga referencia directa al producto o a la calidad, a los precios o los beneficios, o utilizar un nombre creativo que presente una palabra o

por el Ministerio de Agricultura y Desarrollo Rural (Ministerio de Agricultura y Desarrollo Rural, 2014). 
término que lleve un significado especial. Respecto de la forma del logo, hay tres modos básicos que transmiten significados distintos: el círculo, que se relaciona con el movimiento, la perfección y el progreso; el cuadrado, que se relaciona con la experiencia, la robustez y la tradición, y el triángulo que se relaciona con la innovación y el crecimiento (SoyEntrepreneur, 1969). Más allá de las soluciones estéticas, el estilo de redacción de la información, llamado verbal branding, es un aspecto de importancia en el sector de alimentos y bebidas, cuya tendencia se encuentra dirigida hacia un tono coloquial, es decir, nada de letras en mayúsculas llamativas, sino un lenguaje simple, directo y sin pretensiones (Dufranc, 2013). El eslogan (frase breve que comunica de modo impactante una cualidad, característica, beneficio o aspiración de la firma o producto) debe ir acorde también con esta tendencia y debe respetar cinco reglas para ser exitoso: ha de ser corto y simple (3-4 palabras máximo), impactante, fácil de recordar, informar de un beneficio o característica clave y original y exclusivo de su producto para que no se confunda con otro (Ars Logo Design, 2014). El diseño emocional está asociado con el color, así que los consumidores se forman una opinión de los productos en menos de 90 segundos desde su primera interacción con ellos, y entre 62 y 90 \% de esa evaluación está basada solo en el color del producto, por lo que al seleccionar un color para el packaging se debe tener en cuenta el perfil del consumidor, la zona, la clase social, entre otros factores, ya que existe una amplia gama de interpretaciones sobre lo que cada color representa para el consumidor (Pérez, 2009). Según Rojas (2013), los colores tienen las siguientes representaciones: azul: paz, estabilidad, calma, confianza, tranquilidad, sinceridad, afecto, integridad; verde: vida, crecimiento, medio ambiente, salud, dinero, seguridad, relajación, frescura; violeta: realeza, lujo, dignidad, sabiduría, espiritualidad, visión, magia; negro: sofisticación, poder, autoridad, misterio, formalidad, luto; rojo: peligro, pasión, romance, estilo, entusiasmo, audacia, urgencia, energético; naranja: potente, accesible, informal, calidez, entusiasta, apetecible; amarillo: alegría, jovialidad, simpatía, intelecto, energía, calidez, precaución; café: estabilidad, masculinidad, comodidad, confiabilidad, resistencia; blanco: pureza, inocencia, limpieza, delicadeza, refinamiento, simplicidad, y gris: estabilidad, seguridad, carácter, autoridad, madurez. 
Otro componente no menos importante es el diseño de la información, ya que la etiqueta es el elemento visible donde el cliente encontrará toda la información útil que busca: nombre, marca, características (ingredientes, componentes, peso, tamaño...), indicaciones para su uso o conservación, precauciones, nombre del fabricante, procedencia, fecha de fabricación y de vencimiento, entre otros datos de interés que dependen de las leyes o normativas vigentes para cada industria o sector. Cabe anotar que en Colombia la información nutricional es voluntaria hasta diciembre de 2016, cuando pasará a ser obligatoria. El diseño de una etiqueta debe cumplir con las siguientes características: ser adaptable al envase en tamaño, color, forma, entre otros; ser resistente para que perdure desde la salida del producto del almacén hasta llegar a las manos del consumidor final, estar perfectamente adherida al envase; contener la información en el formato exigido por las leyes, normativas o regulaciones del sector; su diseño debe diferenciarlo de otros productos al mismo tiempo que capta la atención del cliente, e incluir datos de contacto, como teléfonos, fax, dirección, sitio web, número de línea gratuita de atención al cliente (Revistaialimentos.com.co, 2011).
El nombre escogido para el producto frutas y verduras solubles fue Cosecha por la connotación que tiene esta palabra con el campo y los productos frescos. El eslogan que se disenó fue "El auténtico sabor del campo", ya que se quería que fuese corto, fácil de recordar, sonoro y descriptivo.

El logo tenía que reflejar un producto natural que evocase los productos tradicionales del campo, pero al mismo tiempo transmitiese progreso, de ahí que el logo fue de forma redonda y su símbolo una cesta de mimbre llena de las frutas o verduras de las que estuviera conformado el producto.

Respecto del color del logo, se aplicaron los colores verde y blanco, ya que transmiten frescura, limpieza, simplicidad y salud (figura 1).

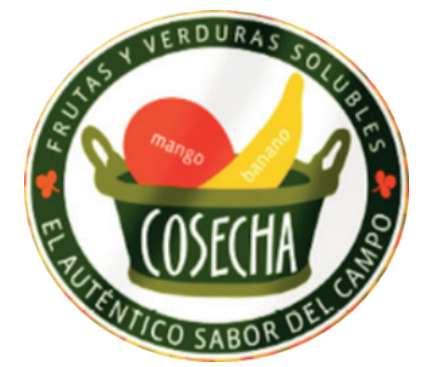

Figura 1. Logo producto Cosecha.

Los colores escogidos para los envases fueron verde, amarillo, naranja, 
rojo y café. Estos colores se escogieron por los atributos que transmiten: color verde: vida, crecimiento, medio ambiente, sanación, dinero, seguridad, relajación, frescura; amarillo: alegría, jovialidad, simpatía, intelecto, energía y calidez; naranja: potente, accesible, informal, calidez, entusiasta y apetecible; rojo: pasión, romance, estilo, entusiasmo, audacia y energético, y café: comodidad, confiabilidad y resistencia.

Estos colores de forma trenzada simulan una textura, como si estuviese hecha de mimbre, que tiene una connotación de tradición de las antiguas canastas con las que se recogían las cosechas.

En el etiquetado se optó por colocar información nutricional, ingredientes, modo de empleo y características excepcionales del producto, como ciento por ciento natural, sin conservantes ni colorantes, sin gluten, un alimento para toda la familia, un alimento para toda ocasión, fácil de preparar y consumir, envase reciclable y reutilizable y no requiere refrigeración.

La funcionalidad es un elemento clave para los consumidores, por tanto se optó por tener envases tanto individuales como familiares, así como para tener en el hogar o portátiles que fueran fáciles de usar, abrir y cerrar.

De acuerdo con esto, se diseñaron los siguientes prototipos de packaging ecológicos para el producto Cosecha: frutas y verduras en polvo soluble. Los diseños se realizaron junto con Alejandro Medina, diseñador gráfico.
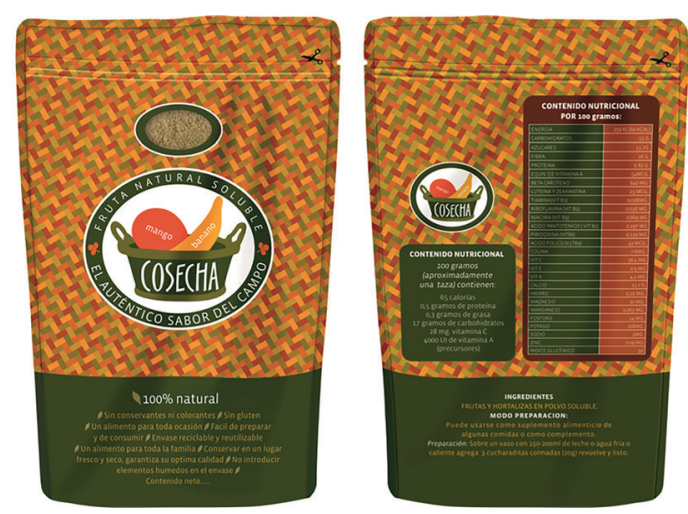

Envase 1: papel reciclado de caña de azúcar y lámina de aluminio Este packaging es reciclable y reutilizable, de tamaño familiar (500 g), con ventana de visualización de contenido, símbolo de tijera que indica por dónde se debe cortar y sistema de cierre tipo Ziploc. 

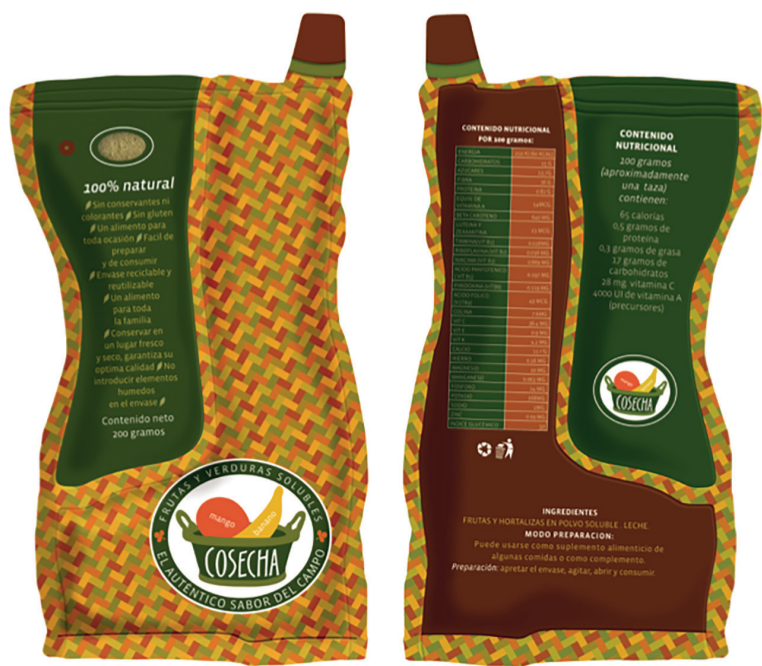

Envase 2: poliácido láctico y lámina de aluminio reciclado Este packaging es biodegradable respecto del poliácido láctico y reciclable del aluminio. Es de tamaño personal (200 ml) para llevar y dispone de un doble compartimento independiente: en uno se encuentra el polvo de frutas y verduras solubles y en el otro el líquido (agua o leche). Para consumirlo, lo único que hay que hacer es apretarlo para que se mezclen ambos contenidos, agitarlo y abrirlo. Dispone de una ventana de visualizacion y un orificio para sentir el aroma del producto.

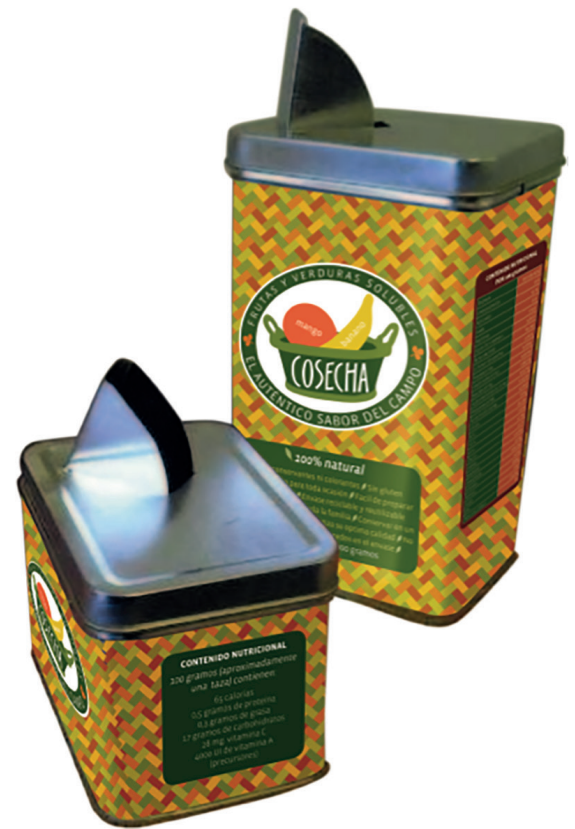

Envase 3: aluminio reciclado

Este packaging es reutilizable y reciclable, tiene dos tamaños: familiar de $900 \mathrm{~g}$ e individual de $450 \mathrm{~g}$. Tiene forma rectangular que facilita el agarre, tapa hermética fácil de abrir y cerrar y dosificador retráctil para no tener necesidad de abrir el envase y poder servirse directamente. 


\section{Conclusiones}

Una vez consultada la muestra de 51 personas pertenecientes a estratos 2 y 3 de Bogotá, se obtuvieron los siguientes resultados:

- Respecto del logo de Cosecha, para la mayoría de los encuestados transmite alegría y añoranza, es fácil de reconocer y les parece que el eslogan "auténtico sabor del campo" es descriptivo $\mathrm{y}$ atractivo.

- Respecto de los beneficios que informa el etiquetado de ser ciento por ciento natural, sin conservantes ni colorantes, sin gluten, un alimento para toda ocasión, fácil de preparar y consumir, envase reciclable y reutilizable y que no requiere refrigeración, la gran mayoría indica que son importantes.

- En cuanto a la semiótica utilizada, para la mayoría fue sencilla y directa, y las palabras utilizadas les sugirieron lo siguiente:

- Cosecha: agricultura y ecología.

- Sabor: gusto.

- Saludable: no contiene elementos artificiales y es satisfactorio.

- Natural: sin elementos artificiales.

- Práctico: sencillo o fácil.
- Conservante: químico.

- Instantáneo: soluble.

- Reciclable: protección al medio ambiente y ecología.

- Jugo: frutas y bebida.

- Familiar: tamaño, hogar, grupo.

- Canasta: compras, antiguo contenedor.

- Calidad: bueno, valor.

- Sostenible: duradero, ecología.

- Mimbre: tradición, recipiente.

- Fresco: reciente, natural.

- Reutilizable: varios usos.

- Colorantes: artificial.

- Aroma: olor, sensación.

- De acuerdo con lo anterior, se deduce que la semiótica utilizada es la correcta, ya que transmite lo deseado.

- Respecto de los aspectos que más llamaron la atención de los prototipos, fueron los materiales con los que estaban hechos, el diseño gráfico, que consideraron llamativo y diferente de los que se encuentran en el mercado.

- Los elementos innovadores y ergonómicos, como la válvula de aroma, la ventana de vista del producto, el envase de llevar de doble compartimento separado, el dosificador retráctil, son los que los motivarían a comprar el producto. 
- La mayoría dijo que compraría el envase de plástico biodegradable hecho a base de fécula de maíz, ya que tiene un diseño innovador, es fácil de llevar y es biodegradable, seguido del envase de aluminio reciclado por su forma rectangular fácil de agarrar, por su dosificador retráctil y su posibilidad de reutilización y reciclaje, y por último el envase hecho de papel reciclado por su fácil apertura y cierre y por ser reutilizable.

\section{Discusión}

- Las decisiones tomadas para el diseño de los envases basados en las teorías de diseño estructural y gráfico fueron adecuadas, pues la mayoría dijo que compraría el producto en cuanto estuviese en el mercado o cuando tuviese más reconocimiento en él mercado.

- El sector del packaging ecológico en Colombia tiene muchas oportunidades, el sector agroindustrial está comenzando a darse cuenta de que, aparte de tener un buen producto, debe saberlo comunicar y presentar en el mercado, a fin de que resulte llamativo para el cliente, y que hay que innovar para poder diferenciarse.

- Se recomienda llevar a cabo un estudio de factibilidad de los envases seleccionados para conocer su coste de elaboración, su posible precio en el mercado y aceptación por parte de los potenciales clientes, a causa de que el precio es un aspecto decisivo para el segmento de mercado.

- Las personas están cada vez más concientizadas del impacto medioambiental que producen los envases y la necesidad del cambio, lo cual abre muchas puertas para seguir desarrollando materiales ecológicos, en su diseño $y$ en conseguir envases que sean certificados como ecológicos.

- La certificación ecológica es voluntaria y no se conocen bases de datos de productos certificados con el sello colombiano ni con sellos de entidades privadas certificadoras, por lo que sería recomendable tener a la mano una base de datos de este tipo y saber si son productos ecológicos o envases ecológicos.

- Es clave seguir innovando en aquellos elementos que le faciliten el uso del producto al consumidor y en la semiótica, ya que 
son elementos importantes a la hora de atraer a clientes.

- No existe información clara de cómo afecta la semiótica a la percepción del cliente según lo que se busque transmitir, por ese motivo sería recomendable realizar un estudio de percepción psicológica del cliente colombiano ante productos.

\section{Referencias bibliográficas}

Aramendiz-Tatis, H., Cardona, C., Jarma, A., Robles, J. y Montalván, R. (2007). Efectos del almacenamiento en la calidad fisiológica de la semilla de berenjena (Solanum melongena L.). Agronomía Colombiana, 25(1), 104-112.

Ardila, I. (2014). Seis tendencias en packaging que marcarán el futuro del envase. Recuperado de Revista P\&M: http://www.revistapym.com.co/destacados/seis-tendencias-packagingque-marcaran-futuro-envase

Ars Logo Design (2014, 20 de mayo). ¿Qué es un slogan? ¿Cómo crear un slogan original y efectivo? Recuperado de http://www.ars-logo-design.com/ es/ar_slogan.htm
Auras, R. A., Lim, L. T., Selke, S. E. y Tsuji, H. (eds.) (2011). Poly (lactic acid): synthesis, structures, properties, processing, and applications. New Jersey: John Wiley \& Sons.

Cajamarca Rueda, A. y Murillo, T. (2011). Packaging: empaques de snacks para el consumidor de bajos ingresos (Tesis de grado, Colegio de Estudios Superiores de Administración, Bogotá, Colombia). Recuperado de http://repository.cesa.edu.co/bitstream/10726/417/2/TG00599.pdf

Cañón, M., Ramírez, A., GutiérrezFranco, E. y López-Ospina, H. A. (2014). Modelo de optimización aplicado a la agroindustria colombiana: plan logístico integrado para la exportación de feijoa fresca. Boletín de Matemáticas, 21(1), 17-31.

Burbano, F. (2013, 27 de agosto). Papel ecológico: ¿qué es un papel ecológico? En Webpapeleria. Recuperado de http://webpapeleria.blogspot. com/2013/08/que-es-un-papel-ecologico-para-que-un.html

Código Visual (2009, 6 de julio). Qué es el packaging. Recuperado de https://codigovisual.wordpress. com/2009/07/06/que-es-el-packaging/ 
Colombia, Ministerio de Agricultura y Desarrollo Territorial (2006, 31 de julio). Resolución 187. Recuperado de http://www.ica.gov.co/getattachment/efc964b6-2ad3-4428-aad5a9f2de5629d3/187.aspx

DANE (2013). Estratificación socioeconómica. Recuperado de http://www. dane.gov.co/index.php/estratificacion-socioeconomica/generalidades

Dufranc, G. (2013, 22 de mayo). Los alimentos saludables cambian el diseño de packaging. En FOROALFA. Recuperado de http://foroalfa.org/ articulos/los-alimentos-saludablescambian-el-diseno-de-packaging

Dussán Sarria, S., Torres León, C. y Reyes-Calvache, P. M. (2014). Efecto del recubrimiento comestible sobre los atributos físico-químicos de mango Tommy Atkins mínimamente procesado y refrigerado. Acta Agronómica, 63(3), 212-221.

Elempaque.com (s. f.). El empaque + coversión. Recuperado de http:// www.elempaque.com/imprimir/Talento-colombiano-en-diseno-de-envases/_4081656

Flórez, O. A., Marín, H. F. y Zapata, J. A. (2009). Estudio de las prácticas de cosecha y poscosecha de la papaya (Carica papaya cv. Maradol) en el departamento del Huila, Colombia. Revista de Investigación Agraria y Ambiental (RIAA), 1, 29-36.

García Mogollón, C., Cury Regino, K. I. y Dussán Sarria, S. (2011). Comportamiento, poscosecha y evaluación de calidad de fruta fresca de guayaba en diferentes condiciones de almacenamiento. Rev. Fac. Nal. Agr., 64(2), 6207-6212.

Incontec Internacional (s. f.). Sello ambiental colombiano. Recuperado de http://www.icontec.org/index. $\mathrm{php} /$ sa/inicio/certificacion-producto/tipos-de-certificados-que-le-puedan-interesar/51-colombia/certificacion-producto/303-sello-ambientalcolombiano

Invima (2014, 7 de julio). Normatividad. Recuperado de https://www.invima.gov.co/index.php?option=com_ content $\&$ view $=$ article\&id $=55 \&$ Item id $=67$

ISO (2005). ISO/TC 122/SC 4 Packaging and the environment. Recuperado de http://www.iso.org/iso/iso_technical_ committee.html\%3Fcommid\%3D52082 
Martín, L. (2013, 32 de enero). Packaging sostenible, cambiar por fuera para cambiar por dentro. En Compromiso Empresarial. Recuperado de http://www.compromisoempresarial.com/carrusel/2013/01/packaging-sostenible-cambiar-por-fuerapara-cambiar-por-dentro/

Mercadeo Sostenible (2012, 25 de octubre). Ecoetiquetas en Colombia. Recuperado de http://www.icesi.edu. co/blogs_estudiantes/mercadeosostenible_stephania_erazo/2012/10/25/ ecoetiquetas-en-colombia/

Merino Sanz, M. J. (2010). Introducción a la investigación de mercados. Madrid: ESIC.

Ministerio de Agricultura de Colombia (2014, 25 de marzo). Autorización a certificadoras de productos ecológicos. Recuperado de https://www. minagricultura.gov.co/tramites-servicios/Paginas/Sello-Ecologico.aspx

Moinhos Rodrigues, J. M., García-Inés, G. S. y Alberto, M. M. (2011, 25 de enero). Envases sostenibles. En ipac.acuicultura. Recuperado de http://www. ipacuicultura.com/noticias/divulgacion/12093/envases_sostenibles.html
Pérez Carballada, C. (2009, 24 de julio). El color del marketing: usando los colores para vender más. Recuperado de http://igomeze.blogspot. com/2009/07/el-color-del-marketingusando-los.html

Pineda, G. (2011, 31 de octubre). Así es el consumidor de bajos recursos. Perfiles de consumo en estratos bajos. Recuperado de http://www.revistapym.com.co/noticias/consumidorpobre/asi-consumidor-bajos-recursos-perfiles-consumo-estratos-bajos ProfesionalRetail (2014, 23 de julio). Seis tendencias en packaging que marcarán el futuro del envase. Recuperado de http://profesionalretail.com/ seis-tendencias-en-packaging-quemarcaran-el-futuro-del-envase/

Revistaialimentos.com.co (2011). El etiquetado de alimentos y el consumidor colombiano. Recuperado de http://www.revistaialimentos.com.co/ ediciones/ediciones-2011/edicion-21/ especial-empaques-y-envases/el-etiquetado-de-alimentos-y-el-consumidor-colombiano.htm

Revista Ambientum (2003, marzo). Generalidades de los envases de brik. Recuperado de http://www.ambientum. 


\section{- Diseño de packaging ecológico - Erika Böhm Silveti y Aida Ximena León Guatame}

com/revista/2003_03/BRIK_imprimir.htm

Revista de Logística (s. f). El vidrio, alternativa ecológica y rentable. Recuperado de http://www.revistadelogistica.com/el-vidrio-alternativaecologica-y-rentable.asp

Rodríguez, N. (2013, 24 de febrero). Infografía creación de una marca. Recuperado de http://nitrodriguez. blogspot.com/2013/02/infografiacreacion-de-una-marca.html

Rojas, J. C. (2013, 30 de mayo). El color en el diseño de empaques. Recuperado de http://www.gerardomuzquiz. com/2013/05/30/el-color-en-el-diseno-de-empaques/

Salazar, J. F. (2011, 1 de noviembre). ¿Cómo los envases metálicos contribuyen al desarrollo sostenible? En EnvaPack. Recuperado de http://www. envapack.com/\%C2\%BFcomo-losenvases-metalicos-contribuyen-aldesarrollo-sostenible/

Selke, S. (1994). Packaging and the environment: alternatives, trends and solutions. Boca Raton, Florida: CRC Press.

SoyEntrepreneur (1969, 31 de diciembre). Define el logo y nombre de tu empresa. Recuperado de http://www. soyentrepreneur.com/define-el-logoy-nombre-de-tu-empresa.html

Twenergy (2011, 27 de diciembre). Los símbolos de reciclaje, ¿qué significan? Recuperado de http://twenergy. com/a/que-significan-los-simbolosde-reciclaje-158

Universia (2014, 19 de septiembre). Según encuesta, colombianos tienen en cuenta el medio ambiente a la hora de decidir su compra. Recuperado de http://noticias.universia.net.co/actualidad/noticia/2014/09/19/1111510/ segun-encuesta-colombianos-cuentamedio-ambiente-hora-decidir-compra.html

Yanhaas (2004, 6 de septiembre). ¡El consumidor colombiano esta cambiando! Recuperado de http://www. yanhaas.com/index.php/yanhaaspoll/item/194-yanhaas-poll-no-26 